Appl. Phys. A 53, 418-421 (1991)

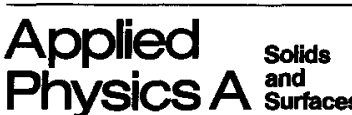

(C) Springer-Verlag 1991

\title{
A New Spin Effect in Photoemission with Unpolarized Light: Experimental Evidence of Spin Polarized Electrons in Normal Emission from Pt(111) and Au(111)
}

\author{
B. Schmiedeskamp, N. Irmer, R. David, and U. Heinzmann \\ Universität Bielefeld, Fakultät für Physik, W-4800 Bielefeld, Fed. Rep. Germany
}

Received 30 April 1991/Accepted 19 July 1991

\begin{abstract}
Unpolarized light ejects spin polarized electrons from $\mathrm{Pt}(111)$ and $\mathrm{Au}(111)$ even if the electron emission occurs normal to the surface. For off normal incidence of $11.8 \mathrm{eV}, 16.9 \mathrm{eV}$, and $21.2 \mathrm{eV}$ radiation, and for the main peaks in the photoemission spectra, a degree of spin polarization of up to $30 \%$ or more is found for the spin polarization component $P_{y}$ perpendicular to the reaction plane. A crystal rotation about its surface normal does not change $P_{y} . P_{y}$ is largest for transitions from bands with symmetry $A_{6}^{3}$. All these experimental findings agree with a recent theoretical prediction [1] of a new spin effect by Tamura and Feder.
\end{abstract}

PACS: $79.60 . \mathrm{Cn}, 71.25 . \mathrm{Pi}$

Spin analysis in the angle- and energy-resolved photoemission is a well established tool to gain a wealth of additional information about the electronic structure of both ferromagnetic [2] and nonmagnetic [4] crystals, surfaces and adsorbates. Most studies on nonmagnetic targets use circularly polarized radiation, since for the photoemission with unpolarized and linearly polarized radiation from non-ferromagnetic centrosymmetric [6] crystals it was for a long time generally believed (see references cited in $[7,8]$ ) that the electrons emitted normal $[9]$ to the surface can only be unpolarized. This belief results from the description of photoemission as a "three-step process": As a consequence of the space inversion symmetry, which the infinite crystal exhibits in contrast to the semi-infinite one, no spin polarization is produced by linearly or unpolarized light in the bulk excitation step. Since transport and perpendicular surface transmission are spin independent, the photoelectrons remain unpolarized $[1,10]$. A theoretical treatment in the "one-step model" [7] and experiment [8] yielded, on the other hand, spin polarized electrons for the special case of normal incidence of linearly polarized radiation and three-fold surfaces. The effect shows the three-fold symmetry in the rotation diagram of the spin polarization, i.e. a sign change when the crystal is rotated about 60 degrees and it was only observed when the time reversed states with $\Lambda_{4}^{3}$ and $\Lambda_{5}^{3}$ symmetry are involved in the transition. The occurrence of spin polarized electrons due to this effect was, however, an exception rather than the rule; up to now the effect was only observed for transitions into the bandgap, i.e. for transitions into evanescent states [8], which emphasizes the surface sensitive nature of this effect.

A spin polarization effect for far more general conditions can be expected if the radiation incidence occurs off normal. This has been predicted in recent theoretical work [1] (again in the one-step model) by Tamura and Feder. Their work deals with normal photoemission with linearly and unpolarized radiation from $\mathrm{Pt}(100)$ (it is in addition stated [1], that the effect should occur for practically every crystal surface): Symmetry arguments show that a spin polarization component perpendicular to the reaction plane defined by photon and photoelectron momenta is not forbidden; the other two components of the spin polarization vector should, however, vanish. The degree of spin polarization is also independent of any rotation of the crystal about the surface normal. Analytical dipole matrix element calculations confirm the conclusions obtained from the symmetry arguments and reveal spin-orbit induced hybridization as its main cause. For our (111)-surfaces this means that the effect is only expected when $\Lambda_{6}^{1} \Lambda_{6}^{3}$-hybrids are involved in the transition [1]. The effect is only predicted for off-normal radiation incidence and vanishes for incidence normal and parallel to the crystal surface.

This work reports on the experimental verification of this spin polarization effect with unpolarized radiation for the case of the three-fold surfaces Pt(111) and $\mathrm{Au}(111)$.

The experimental set-up is shown in Fig. 1. Unpolarized radiation from a discharge tube hits the crystal surface at an angle of $62^{\circ}$ with respect to the surface 


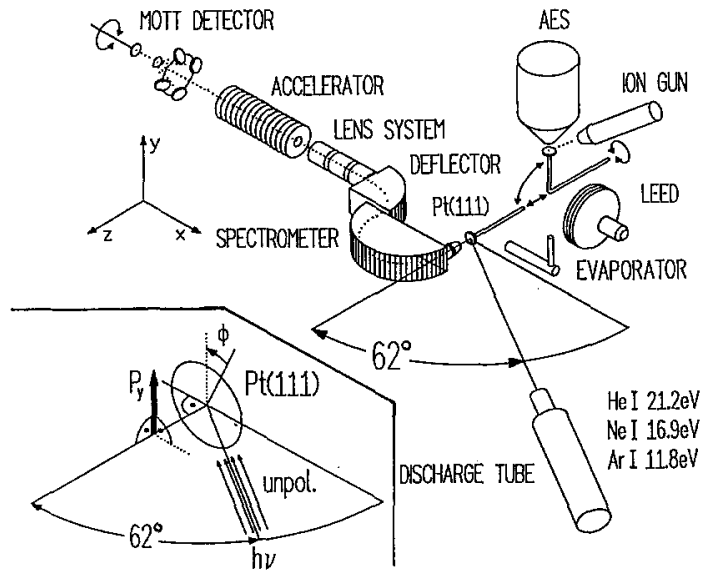

Fig. 1. Experimental set up

normal. The energy of the normally emitted electrons is determined with an electron spectrometer. Two components of the spin polarization vector are obtained by Mott scattering: $P_{y}$ is perpendicular to the reaction plane, $P_{z}$ is parallel to the crystal surface normal and turned out to be zero for all measurements given in this work. The Pt(111) surface was prepared with sputtering and heating in oxygen [11], Au(111) measurements were performed with a thick epitaxial Au layer on $\mathrm{Pt}(111)$ which was deposited with a resistively heated Au evaporator [12]. The surfaces were characterized by Auger electron spectroscopy (AES) and LEED.

Figure 2 presents the experimental results obtained for $\mathrm{Au}(111)$ and $\mathrm{Pt}(111)$ and a photon energy of $21.2 \mathrm{eV}$ (He I). Photoemission spectra are given in the left part. Dots together with the solid line denote the total (nonspin resolved) intensity $I$. Below those curves the partial (spin resolved) intensities $I_{+}$(solid curve) and $I_{-}$(dotted curve) are given. They are obtained from $I$ and the measured spin polarization $P_{y}$ by means of $I_{+}=\frac{I}{2}\left(1+P_{y}\right)$ and $I_{-}=\frac{I}{2}\left(1-P_{y}\right)$ and denote the intensities for spin up and spin down with respect to the reaction plane, respectively. In both of the spectra we find parts of the photoemission spectra with a considerable degree of spin polarization. For $\operatorname{Pt}(111)$ the degree is about $20 \%$ for the first peak below $E_{\mathrm{F}}$ while the electrons contributing to the second peak turn out to be unpolarized. For $\mathrm{Au}(111)$ we find two peaks with a slightly lower degree of spin polarization and opposite spin polarization signs.

That the mechanism reported earlier in $[7,8]$ to create spin polarized photoelectrons by means of linearly polarized radiation and the new mechanism which works even with unpolarized radiation (predicted in [1] and verified in this paper) are not identical is obvious from the dependence of $P_{y}$ on a rotation $\phi$ of the crystal about the surface normal. In the former case $P_{y}$ should show a sinusoidal dependence on $\phi$ with a change of sign when the sample is rotated by $60^{\circ}$. For the latter (new) effect Tamura and Feder predict no change of $P_{y}$ with $\phi$. The dependence of $\boldsymbol{P}_{y}$ on the crystal rotation angle $\phi$ has therefore been determined and is displayed in the right part of Fig. 2. The studies were performed for all three peaks in the photoemission spectra which yield a con-

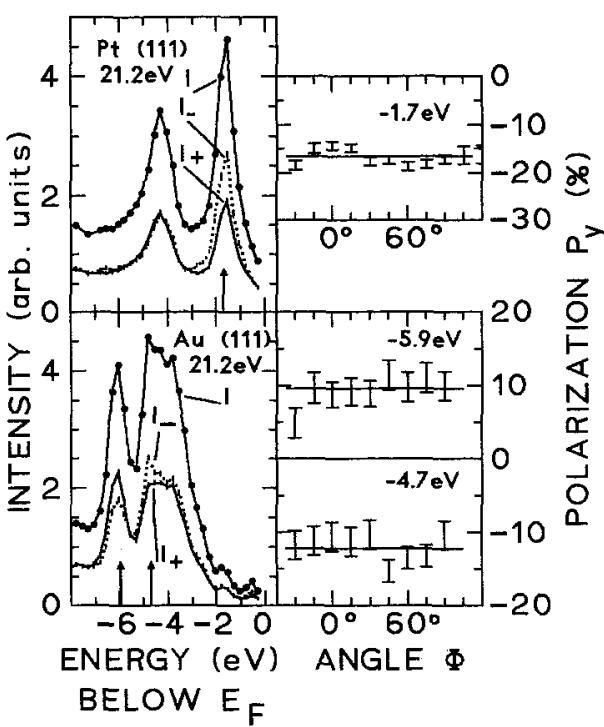

Fig. 2. Left: Spin-resolved spectra for normal photoemission with unpolarized radiation from $\mathrm{Pt}(111)$ and $\mathrm{Au}(111)$. I denotes the total (non spin resolved) intensity, $I_{+}$and $I_{-}$are the partial (spin resolved) intensities. Arrows mark the energies at which the dependence of the spin polarization on the angle $\phi$ was measured. The small dashes in $I_{+}$and $I_{-}$represent the total experimental error which includes the statistical error of the count rates and the uncertainty of the detector asymmetry function. Right: Dependence of the spin polarization component $P_{y}$ on the rotation $\phi$ of the crystal surface about its surface normal. Within the experimental accuracy no significant variation is observed for Au. Only a slight dependence of $P_{y}$ on $\phi$ is found for $\mathrm{Pt}(111)$

siderable degree of spin polarization. The corresponding energetic positions are marked in the left part of Fig. 2 by arrows. In all cases the spin polarization $P_{y}$ shows hardly any change with $\phi$. Although a slight systematic variation of $P_{y}$ with $\phi$ seems to exist for Pt(111) the main part of the spin polarization is invariant in the rotation diagrams and thus provides experimental evidence for the new spin polarization effect. Corresponding spectra were also measured for $16.9 \mathrm{eV}$ and $11.8 \mathrm{eV}$ photon energy. They are displayed in the left part of Fig. 3 and show even higher spin polarization values than the results in Fig. 2.

A further point concerns the mechanism that produces the spin polarization. Following the prediction [1] spinorbit induced hybridization of $A_{6}^{1}$ and $\Lambda_{6}^{3}$ bands must be contained in either the occupied or the unoccupied bands which are involved in the transition. In order to get further information about this we discuss the results together with a bandstructure of $\mathrm{Pt}$ in the $A$-direction which is given in the right part of Fig. 3. The bandstructure is a calculation of Eckardt and Noffke [13]. The calculations also yield the spatial parts which contribute to the individual bands. Bands with $\Lambda_{6}^{1}$-symmetry $\left(\Lambda_{1}\right.$ spatial part dominating) are represented by the heavy solid lines. Bands with symmetry $A_{6}^{3}$ (dominating $\Lambda_{3}$ spatial part) by dots. Thin lines represent bands with symmetry $A_{45}^{3}$. They should only contribute to the spin polarization if transitions occur into $A_{6}^{1} A_{6}^{3}$ hybrids [1]. The arrows in the bandstructure of Fig. 3 indicate the transitions which contribute to the Pt spectra in Figs. 2 and 3. 

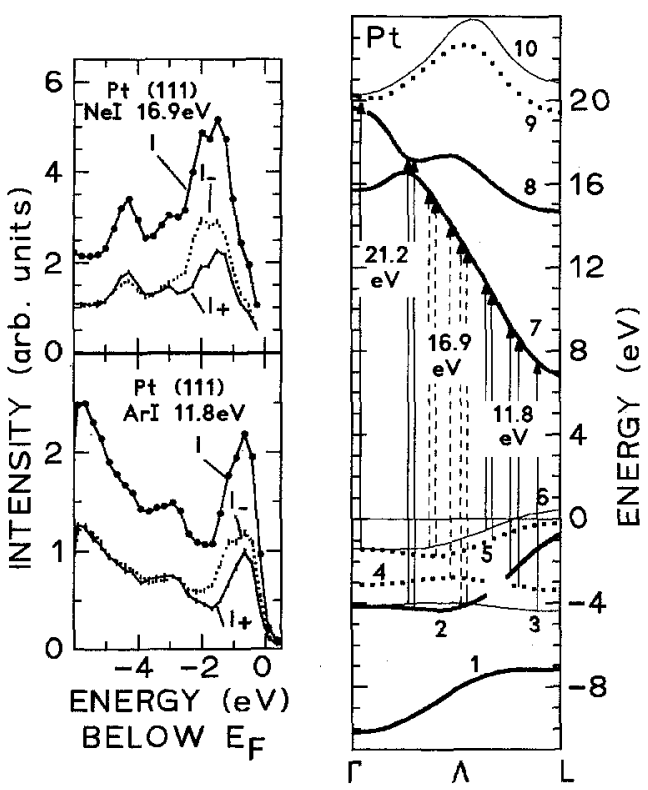

Fig. 3. Left: Spin-resolved spectra for normal photoemission with unpolarized radiation from $\mathrm{Pt}(111)$. The meaning of the symbols is identical to that in Fig. 2. Right: Bandstructure of Pt in the A-direction. The bandstructure is from Eckardt and Noffke [13].

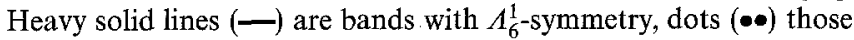
with $\boldsymbol{A}_{6}^{3}$-symmetry. Thin solid lines are bands with $A_{45}^{3}$-symmetry. The arrows represent the transitions which contribute to the spectra for $\mathrm{Pt}(111)$ in Figs. 2 and 3

For $21.2 \mathrm{eV}$ photon energy spin polarization is only obtained for the peak at $1.5 \mathrm{eV}$ below $E_{\mathrm{F}}$ which is due to a transition from the almost degenerate spin-orbit split $A_{45}^{3} A_{6}^{3}$ band pair near $\Gamma$ into the band gap near $20 \mathrm{eV}$ final energy. The bandstructure calculation yields $\Lambda_{6}^{1} \Lambda_{6}^{3}$ hybridization for both of these areas of the bandstructure. There is thus no hint whether the spin polarization is due to hybridization in the occupied or the unoccupied part of the bandstructure and we also get no information about whether $A_{6}^{3}$ or $A_{45}^{3}$ bands are more strongly involved in the production of the spin polarization. For 16.9 and $11.8 \mathrm{eV}$ photon energy the main peak in the photoemission spectra is due to transitions from the spin orbit split $A_{45}^{3} \Lambda_{6}^{3}$ band pair near $E_{\mathrm{F}}$ into the free-electronlike final band in the middle of the $A$-direction and close to $L$, respectively. In this part of the bandstructure the unoccupied bands have almost pure $\Lambda_{6}^{1}$-symmetry [13]. The spin polarization can therefore only be due to hybridization of the occupied bands. Regarding again the spectra in the left part of Fig. 3 we note that the spin polarization is located in the left part of the peak and is therefore at least mainly (if not exclusively) due to the transition from the $A_{6}^{3}$-band and not from the $A_{45}^{3}$-band. Since this upper $A_{6}^{3}$-band also contains a considerable amount of the $\Lambda_{1}$ spatial part [13] the experimental data support strongly the prediction that in the present case the spin polarization is due to $A_{6}^{1} A_{6}^{3}$ hybridization of the occupied bands.

Finally we note some striking similarities between this new effect and a well-known spin polarization result in photoionization [14] and discuss them in order to gain a further understanding for the mechanism that produces the spin polarization. Following the prediction [1] $P_{y}$ should scale as

$P_{y} \propto \sin 2 \Theta \operatorname{Im}\left\{m_{\|} m_{\perp}^{*}\right\}$

with $m_{\|}$and $m_{\perp}$ being the transition matrix elements for excitations with the $\|-$ and $\perp$-component of the $\mathbf{E}$ vector of the incident radiation and $\Theta$ the incidence angle of the radiation with respect to the surface normal. The photoionization of free atoms and molecules with unpolarized radiation can also result in spin polarized electrons if the electron emission occurs at a non-zero angle $\Theta$ with respect to the direction of the incident radiation [14]. $\mathbf{P}$ is then also perpendicular to the reaction plane and

$|\mathbf{P}| \propto \sin 2 \Theta \operatorname{Im}\left\{D_{1} D_{2}^{*}\right\}$,

i.e. the polarization scales also with the product of two transition matrix elements. $D_{1}$ and $D_{2}$ are the transition matrix elements for transitions from one initial state into two different but energetically degenerate continuum states [14]. Spin polarization is due to a quantum mechanical interference of these continuum waves and only yields a non-zero value of $\operatorname{Im}\left\{D_{1} D_{2}^{*}\right\}$ if a phase difference exists between the two waves [14]. In addition the spin polarization sign is opposite for transitions from spinorbit-split initial states, demonstrating that the spin-orbit interaction is responsible for the spin polarization. The physical mechanism that produces the spin polarization $P_{y}$ seems to be closely related to that in photoionization. This is firstly supported by the almost identical description by equations (1) and (2) and secondly by the necessity of spin-orbit interaction for both effects.

Summarising, spin polarized electrons were obtained in the photoemission with unpolarized light from $\mathrm{Pt}(111)$ and $\mathrm{Au}(111)$ even for normal electron emission. A degree of spin polarization of up to more than $30 \%$ is found for the component $P_{y}$ perpendicular to the reaction plane. $P_{y}$ is almost independent of a rotation of the crystal about the surface normal and turns out to be a maximum for transitions from bands with $\Lambda_{6}^{3}$-symmetry. All these findings strongly support the assertion that this new spin polarization effect for (111) surfaces is due to the same mechanism as that predicted recently by Tamura and Feder [1]. Similarities also exist with a corresponding effect in photoionization of free atoms and molecules where the existence of spin polarization requires spin-orbit interaction and phase differences between final state wave functions. In view of the general occurrence of spin polarization for different targets and photon energies and in view of the high spin polarization values obtained, it is worth noting that the effect can also be present in the photoemission from other nonmagnetic and ferromagnetic crystals.

Acknowledgements. We are indebted to E. Tamura and R. Feder for providing their theoretical results prior to publication. Thanks are due to J. Noffke for supplying the details about the spatial parts of the Pt bandstructure calculation. Intensive discussions with R. Feder, E. Tamura, and J. Noffke are acknowledged. The work was financially supported by the Deutsche Forschungsgemeinschaft (SFB 216). 


\section{References}

1. E. Tamura, R. Feder: Submitted for publication and private communications

2. E. Kisker: In Ref. [3], Chap. 12 and references therein

R. Feder: In Ref. [3], Chap. 4 and references therein

3. R. Feder (ed.): Polarized Electrons in Surface Physics (World Scientific, Singapore 1985)

4. U. Heinzmann: Phys. Script. T 17, 77-88 (1987) and references therein

F. Meier, D. Pescia: In Ref. [5], Chap. 7

M. Wöhlecke, G. Borstel: In Ref. [5], Chap. 10

J. Kirschner: Polarized Electrons at Surfaces (Springer, Berlin, Heidelberg 1985)

5. F. Meier, B.P. Zakharchenya (eds.): Optical Orientation, Modern Problems in Condensed Matter Sciences, Vol. 8 (North-Holland, Amsterdam 1984)

6. For non-centrosymmetric surfaces a small spin-polarization can arise via a mechanism described by S.F. Alvarado, H. Riechert, N.E. Christensen: Phys. Rev. Lett. 55, 2716-2719 (1985)

7. E. Tamura, W. Piepke, R. Feder: Phys. Rev. Lett. 59, 934-937 (1987)

8. B. Schmiedeskamp, B. Vogt, U. Heinzmann: Phys. Rev. Lett. 60, 651-654 (1988)
9. For off-normal photoemission spin polarization can be due to spin-dependent diffraction or phase matching conditions in the surface transmission step: J. Kirschner, R. Feder, J.F. Wendelken: Phys. Rev. Lett. 47, 614-617 (1981)

10. R. Feder: In Ref. [3], Chap. 4, pp. 215, 219, 223

11. A. Eyers, F. Schäfers, G. Schönhense, U. Heinzmann, H.P. Oepen, K. Hünlich, J. Kirschner, G. Borstel: Phys. Rev. Lett. 52, 1559-1562 (1984)

12. B. Vogt, B. Schmiedeskamp, U. Heinzmann: Z. Phys. B 80, 359-364 (1990)

13. The bandstructure is displayed in: G. Leschik, R. Courths, H. Wern, S. Hüfner, H. Eckardt, J. Noffke: Solid State Commun. 52, 221-225 (1984); the calculations were performed along the lines described in: L. Fritsche, J. Noffke, H. Eckardt: J. Phys. F 17, 943-965 (1987)

The unoccupied bands are shifted by $0.75 \mathrm{eV}$ towards higher energies as reported by H. Wern, R. Courths, G. Leschik, S. Hiufner: Z. Phys. B 60, 293-310 (1985). The spatial parts of the individual bands were kindly supplied by J. Noffke

14. U. Heinzmann: In Fundamental Processes in Atomic Collision Physics, ed. by H. Kleinpoppen, J.S. Briggs, H.O. Lutz (Plenum, New York 1985) pp. 269-293

U. Heinzmann, G. Schönhense, J. Kessler: Phys. Rev. Lett. 42, 1603-1605 (1979)

G. Schönhense, V. Dzidzonou, S. Kaesdorf, U. Heinzmann: Phys. Rev. Lett. 52, 811-814. (1984) 